\section{A) Check for updates}

Cite this: Polym. Chem., 2019, 10 1626

\title{
Orthogonally clickable hyperbranched polymers: effect of reactant size and polarity on core- functionalization of peripherally jacketed HBPs $\uparrow$
}

\author{
Suresh Kumar Perala and S. Ramakrishnan (iD)*
}

\begin{abstract}
We describe a strategy for the preparation of an "orthogonally clickable" HB polyester (HBP) via a melt trans-esterification process, starting from a suitably designed $\mathrm{AB}_{2}$ monomer. The resulting $\mathrm{HBP}$ carries an allyl group on each repeat unit within the core-region and numerous propargyl groups on the molecular periphery; selective reaction of the periphery, to yield jacketed HBPs, permitted the examination of various factors that govern the access to internal reactive (allyl) sites. First, either hydrophobic docosyl (C22) or hydrophilic PEG350 segments were quantitatively clicked onto the periphery via the azide-yne reaction; subsequently, access of the internal allyl groups to thiol-ene reaction was examined using organic thiols of varying size and polarity. It was seen that the extent of reaction depends not only on the size of the thiol reactant but also on its relative polarity; for instance, a polar thiol is less effective in accessing the internal allyl groups of a HBP bearing docosyl segments on the periphery when compared to a non-polar thiol of roughly the same size. Likewise, when PEG segments are installed at the periphery, access to more polar reactants is favoured when compared to non-polar analogues of a similar size. These studies provide some interesting insights that could be useful in designing HBP supported catalysts for substrate-selective transformations.
\end{abstract}

Received 20th October 2018 Accepted 17th February 2019

DOI: $10.1039 /$ c8py01499j

rsc.li/polymers branched systems. Core reactive sites of compact HBPs are often difficult to fully react, ${ }^{19-23}$ whereas the peripheral functional groups can usually be modified quantitively. ${ }^{13-15}$ On the other hand, in the case of segmented HBPs, both core and peripheral reactive sites are readily transformed quantitatively because of the low branching density of the parent $\mathrm{HBP}^{24,25}$ Gao and co-workers synthesized a range of segmented HBPs bearing amino groups in the backbone; these were readily modified by Menshutkin reaction, which permitted them to incorporate a variety of functional groups, such as alkynyl, azido, hydroxyl, carboxyl and so forth. ${ }^{7}$ Some years ago, we reported the synthesis of orthogonally clickable HBPs which carries only a single propargyl group at the core and numerous allyl groups at the periphery; ${ }^{26}$ these were then functionalized with two different chromophores (a FRET donor-acceptor pair) using the alkyne-azide and thiol-ene click reactions, respectively, in order to study the energy transfer process. HBPs carrying a single catalytic core site have also been examined as synthetic enzyme-mimics with reasonable success. ${ }^{27}$

Peripherally clickable HBPs provide excellent opportunities to place multiple segments on the periphery of a globular polymer structure, which could provide interesting avenues for exploration. Some years ago, we showed that when two immiscible segments, such as PEG and long-chain alkyl segments, are randomly installed on the periphery in a single step, the 
backbone of the HBP reconfigures so as to segregate the two segments and form Janus structures. ${ }^{15}$ Likewise, when the peripheral groups are randomly functionalized with three types of immiscible segments, the HBPs self-segregate to form Tripodal structures. ${ }^{16}$ Earlier studies had also demonstrated the reorganization of core-shell type dendritic polymers, when dispensed at the air-water interface, that causes the hydrophilic branched backbone to effectively flatten and the hydrophobic segments to collocate above the water surface; ${ }^{28-31}$ further, SAXS studies confirmed that such core-shell segregation occurs even in the bulk, and thereby forming a lamellar morphology. ${ }^{30}$

Thus, whereas a variety of hetero-functionalized HBPs have been studied, a systematic study of the factors that govern the extent of functionalization of the internal core-region has not been reported. It is relevant to recall here the efforts of Ellis and Twyman concerning the effect of molecular crowding on the access to a single core-catalytic site in suitably designed HBPs; they showed that both positive and negative dendritic effect can be observed as a function of molecular weight of the $\mathrm{HBP}^{32}$ In the present study, we first describe the synthesis and functionalization of an orthogonally clickable HBP that carries allyl groups on every repeat unit and propargyl groups on the periphery. We then explore the various factors that govern the accessibility of the core allyl groups after the periphery of the HBP is jacketed with either hydrophilic PEG segments or hydrophobic docosyl (C-22) segments.

\section{Experimental section}

\section{Materials and methods}

5-Hydroxyisophthalic acid, $\mathrm{NaH}$, 1-octane thiol, propargyl bromide (80 wt\% in toluene), $\mathrm{LiAlH}_{4}, \mathrm{DBTDL}$ (dibutyl tin dilaurate), PEG-350 monomethylether, tetraethylene glycol monomethylether, hexadecane thiol, docosanol, tosyl chloride, thioglycerol and 2-mercaptoethanol were purchased from Sigma-Aldrich chemical company and used as such. All other chemicals were procured from other commercial sources (AlfaAesar, Spectrochem, SDFCL, Nice chemicals) and used directly, unless mentioned otherwise. All organic solvents were procured from Fischer Scientific and/or SDFCL chemicals; they were dried and distilled, if required. ${ }^{1} \mathrm{H}$-NMR spectra were recorded on a Bruker AV $400 \mathrm{MHz}$ spectrometer using suitable deuterated solvents and TMS as internal reference. Gel Permeation Chromatography (GPC) measurements were performed using a Waters GPC system with R.I. detector and THF was used as the eluent. The detailed synthesis procedures for the monomers and their NMR spectral data are provided in the ESI. $\dagger$

\section{Synthesis of $\mathrm{AB}_{2}$ monomer}

5-((2-(Hydroxymethyl)pent-4-en-1-yl)oxy)isophthalic acid (1.2 g, $4.3 \mathrm{mmol}), \mathrm{NaHCO}_{3}(1.08 \mathrm{~g}, 12.9 \mathrm{mmol})$ were taken in DMSO $(20 \mathrm{~mL})$ and it was stirred for $1 \mathrm{~h}$ at room temperature under nitrogen atmosphere. Propargyl bromide (1.27 g, $10.75 \mathrm{mmol}$ ) was added to the reaction mixture. After $24 \mathrm{~h}, \mathrm{CHCl}_{3}$ was added to it, and it was washed a few times with water to remove DMSO and salts. The $\mathrm{CHCl}_{3}$ layer was passed through $\mathrm{Na}_{2} \mathrm{SO}_{4}$ and concentrated under reduced pressure to yield the product as a light brownish color viscous liquid. This was further purified using silica gel column chromatography using EtOAc and pet ether $(10: 90)$ as the eluent. Yield $=87 \%$. ESI-MS of the monomer (Fig. S27†) confirmed the structure of the monomer.

${ }^{1} \mathrm{H}-\mathrm{NMR}$ (400 MHz, $\left.\mathrm{CDCl}_{3}, \delta \mathrm{ppm}\right): 2.14-2.20(\mathrm{~m}, 1 \mathrm{H}$, $\mathrm{CH}_{2} \mathrm{CHCH}_{2} \mathrm{CH}-$ ); 2.28-2.31 (q, 2H, $\mathrm{CH}_{2} \mathrm{CHCH}_{2} \mathrm{CH}-$ ); 2.57-2.58 (t, 2H, $\left.-\mathrm{OCH}_{2} \mathrm{CCH}\right) ; 3.79-3.83$ (m, 2H, $\left.\mathrm{HOCH}_{2} \mathrm{CH}-\right)$; 4.12-4.14 (q, 2H, $\left.\operatorname{ArOCH}_{2} \mathrm{CH}-\right)$; 4.97-4.98 (d, 2H, - $\left.\mathrm{OCH}_{2} \mathrm{CCH}\right) ; 5.10-5.16$ (m, 2H, $\left.\mathbf{C H}_{2} \mathrm{CHCH}_{2} \mathrm{CH}-\right)$; 5.82-5.92 (m, $1 \mathrm{H}, \mathrm{CH}_{2} \mathbf{C H C H}_{2} \mathrm{CH}-$ ); 7.82 (d, 2H, -ArH); 8.35 (s, 1H, -ArH).

\section{Preparation of parent HBP}

The polymerization was done by melt-transesterification polycondensation method. ${ }^{13}$ The $\mathrm{AB}_{2}$ monomer $(550 \mathrm{mg}$, $1.54 \mathrm{mmol})$, dibutyl tin dilaurate $(19.5 \mathrm{mg}, 0.03 \mathrm{mmol})$ and a spin bar were taken in a small test tube-like flask with a bulb at the bottom; and the contents were heated at $120{ }^{\circ} \mathrm{C}$, with stirring, under dry nitrogen purge. Upon increase in viscosity (spin-bar stopped spinning), the flask was heated to $150{ }^{\circ} \mathrm{C}$ using Kugelrohr apparatus under reduced pressure ( $\sim 5 \mathrm{mbar})$, with constant rotation. Bubbling of the melt revealed the formation of condensate (propargyl alcohol), which was continuously removed. Once the melt become highly viscous, the reaction was stopped, and the contents were cooled to room temperature. The polymer was dissolved in $\mathrm{CHCl}_{3}$ and precipitated out in $\mathrm{CH}_{3} \mathrm{OH}$; it was once again dissolved in $\mathrm{CHCl}_{3}$ and precipitated in petroleum ether to obtain a light-brownish fibrous solid. Yield $=85 \%$; the molecular weight $\left(M_{\mathrm{n}}\right)$ of the HBP, estimated by GPC (standard calibration using PS standards) was around $3200 \mathrm{~g} \mathrm{~mol}^{-1}$ and the PDI was 3.7 (Fig. S21 $\dagger$ ).

${ }^{1} \mathrm{H}-\mathrm{NMR} \quad\left(400 \mathrm{MHz}, \quad \mathrm{CDCl}_{3}, \quad \delta \mathrm{ppm}\right): 2.36 \quad(2 \mathrm{H}$, $\left.\mathrm{CH}_{2} \mathrm{CHCH}_{2} \mathrm{CH}-\right) ; 2.37$ (2H, $\left.\mathrm{CH}_{2} \mathrm{CHCH}_{2} \mathrm{CH}-\right) ; 2.53$ (1H, $\left.-\mathrm{CO}_{2} \mathrm{CH}_{2} \mathrm{CCH}\right) ; \quad 4.1 \quad\left(2 \mathrm{H}, \quad \mathrm{ArOCH}_{2} \mathrm{CH}-\right) ; 4.46 \quad(2 \mathrm{H}$, $\left.\mathrm{ArOCH}_{2} \mathrm{CHCH}_{2} \mathrm{O}-\right) ; 4.91\left(2 \mathrm{H},-{ }_{-} \mathrm{CO}_{2} \mathbf{C H}_{2} \mathrm{CCH}\right) ; 5.09(2 \mathrm{H}$, $\left.\mathbf{C H}_{2} \mathrm{CHCH}_{2} \mathrm{CH}-\right)$; 5.83 (1H, $\left.\mathrm{CH}_{2} \mathbf{C H C H}_{2} \mathrm{CH}-\right) ; 7.74$ (2H, -ArH); $8.24(1 \mathrm{H},-\mathbf{A r H})$.

\section{Alkyne-azide click reaction of the peripheral propargyl groups}

Preparation of HBP-DOCO. The parent HBP (200 mg, $0.666 \mathrm{mmol}$ of repeat unit) and docosyl azide (257 mg, $0.733 \mathrm{mmol}$ ) were taken in a round-bottom flask and dissolved in THF; dry-nitrogen was purged for $10 \mathrm{~min}$. $\mathrm{CuSO}_{4} \cdot 5 \mathrm{H}_{2} \mathrm{O}$ (8.3 $\mathrm{mg}, 0.0333 \mathrm{mmol}$ ) and sodium ascorbate $(13.1 \mathrm{mg}$, $0.066 \mathrm{mmol}$ ) were dissolved in a small amount of water and added to the reaction vessel; dry-nitrogen purge was continued for additional $10 \mathrm{~min}$. The flask was then sealed with a cap and the contents were stirred for 3 days at $50{ }^{\circ} \mathrm{C}$. The reaction mixture was concentrated under reduced pressure and precipitated in cold pet ether, followed by wash with diethyl ether to get a light pink powder polymer. Yield $=91 \% ; M_{\mathrm{n}}$ value by GPC was found to be around $4300 \mathrm{~g} \mathrm{~mol}^{-1}$ with a PDI of 4.8 (Fig. S23†). 
HBP-PEG350 was prepared using the same procedure to yield the polymer in $83 \%$ yield; $M_{\mathrm{n}}$ value by GPC was found to be around $4800 \mathrm{~g} \mathrm{~mol}^{-1}$ with a PDI of 2.3 (Fig. S22 $\dagger$ ). In this case, the concentrated reaction mixture was precipitated in cold diethyl ether to get the product.

General procedure for thiol-ene click reactions. The peripherally functionalized HBP (1 equiv.), required thiol (5 equiv.), 2,2-dimethoxy-2-phenylacetophenone (photo-initiator) (0.18 equiv.) were dissolved in $\mathrm{CHCl}_{3}$ and taken in a Pyrex tube, which was placed on a rotating carrousel in a photoirradiation chamber; the contents were irradiated with UV light (365 nm) for $10 \mathrm{~h}$ at room temperature. The solvent was removed under reduced pressure and the residue was washed with a nonsolvent, like $\mathrm{CH}_{3} \mathrm{OH}$ /pet ether/diethyl ether, depending on the solubility of the resulting polymer.

For comparative studies, the HBP solution in chloroform (30 $\mathrm{mg} \mathrm{mL}^{-1}$ ) is taken together with the desired thiol, in the required quantity, along with the photoinitiator, and placed in the carrousel; to ensure identical reaction conditions, all other reactant solutions carrying the same number of equivalents of different thiols and the HBP, were placed in different slots on the same carrousel. All samples were then irradiated together under constant rotation in the photoreactor using light of $\sim 365 \mathrm{~nm}$ wavelength for $10 \mathrm{~h}$. The clicked polymer samples were isolated by removal of the solvent followed by washing with $\mathrm{CH}_{3} \mathrm{OH} /$ pet ether/diethyl ether to remove any excess unreacted thiol, followed by analysis by NMR to estimate the extent of reaction.

\section{Results and discussion}

The $\mathrm{AB}_{2}$ type monomer carrying an allyl unit in the spacer segment and two propargyl ester groups was prepared starting from 5-hydroxyisophthalic acid (as shown in Scheme 1); the allyl group bearing spacer was prepared from commercially available diethylmalonate, as described in the ESI. $\dagger$ The $\mathrm{AB}_{2}$ monomer was polymerized by melt transesterification polycondensation process using dibutyltin dilaurate (DBTDL) as the catalyst at $150{ }^{\circ} \mathrm{C}$, with continuous removal of the condensate, propargyl alcohol; this yields the orthogonally clickable HBP bearing allyl groups on every repeat unit within the core-region and propargyl groups at the periphery (Scheme 2).

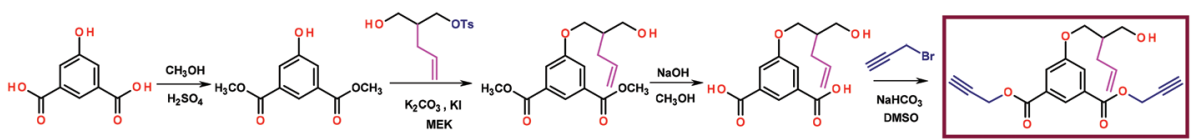

Scheme 1 Preparation of allyl and propargyl groups bearing $\mathrm{AB}_{2}$ monomer.

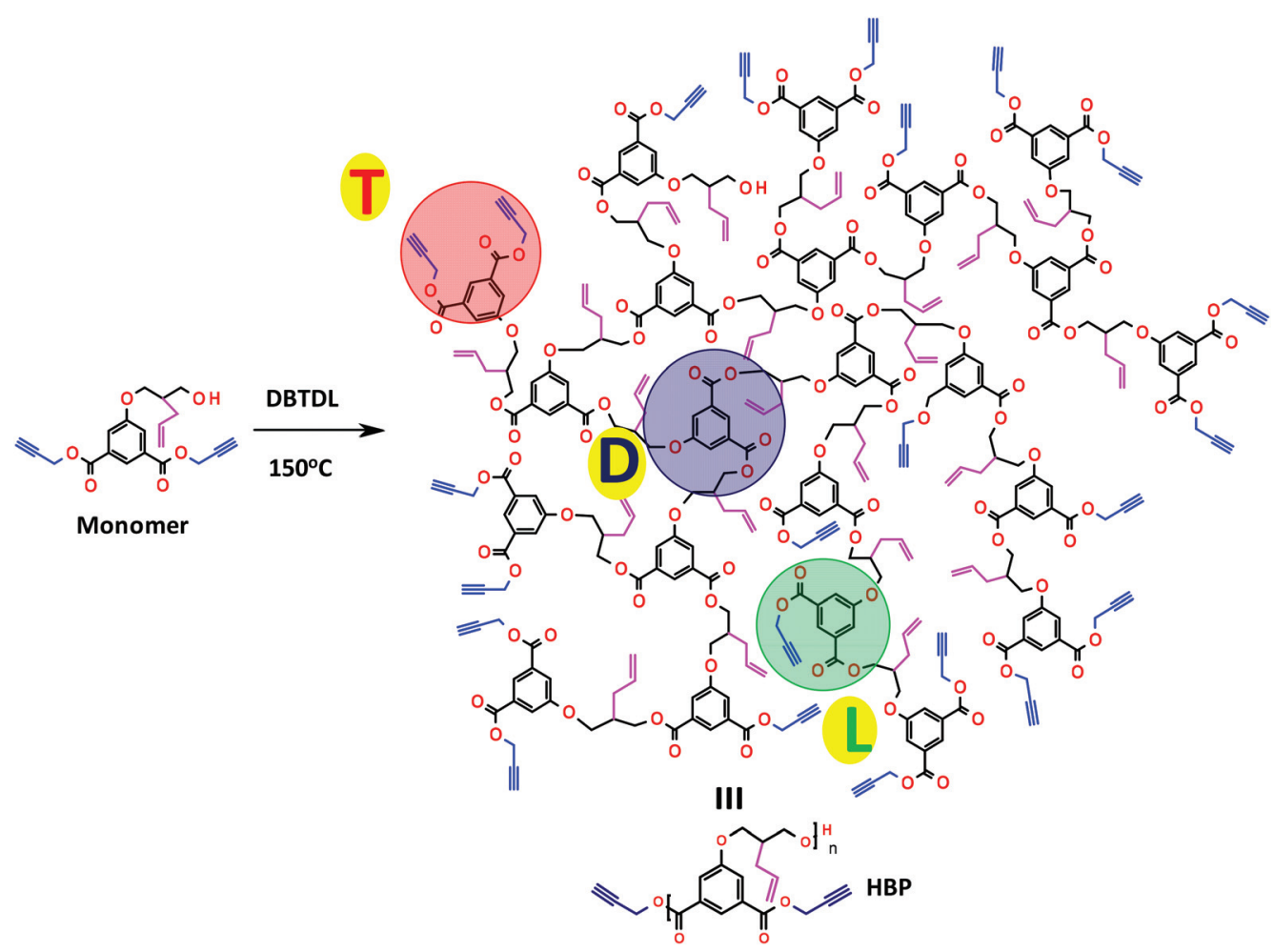

Scheme 2 Preparation of orthogonally clickable HBP bearing core-allyl and peripheral propargyl groups. Typically, HBPs consist of 3 types of units, namely dendritic $(D)$, linear $(L)$ and terminal $(T)$, as depicted in the structure. 
The polymerization was first done under dry $\mathrm{N}_{2}$ purge, followed by a few hours under reduced pressure using Kugelrohr apparatus. ${ }^{13}$ The HBP was characterized by ${ }^{1} \mathrm{H}-\mathrm{NMR}$ spectroscopy; the ${ }^{1} \mathrm{H}-\mathrm{NMR}$ spectra of the monomer and HBP are shown in Fig. 1. From the NMR spectra, it is evident that the core allyl groups remain intact during the melt polymerization at $150{ }^{\circ} \mathrm{C}$, whereas the intensity of the propargyl ester groups decreased to half its original value as reflected by the relative intensities of the relevant peaks in the monomer and HBP. The molecular weight of the HBP was determined by gel permeation chromatography, and the $M_{\mathrm{n}}$ and PDI were found to be $3200 \mathrm{~g} \mathrm{~mol}^{-1}$ and 3.7, respectively (Fig. S21†); the high dispersity is typical of HBPs, where the distribution gets wider with percent conversion.

\section{Selective functionalization of the periphery of the HBP}

The peripheral propargyl groups of the HBP were first functionalized with hydrophilic PEG350 (polyethylene glycol 350, monomethylether mono-azide) under standard alkyne-azide click reaction conditions ${ }^{33}$ in the presence of $\mathrm{CuSO}_{4} \cdot 5 \mathrm{H}_{2} \mathrm{O}$ / sodium ascorbate (Scheme 3). The ${ }^{1} \mathrm{H}-\mathrm{NMR}$ spectrum reveals quantitative conversion; the complete disappearance of propargyl ester protons peaks at around $2.5 \mathrm{ppm}$, the appearance of the triazole proton peak at around $7.9 \mathrm{ppm}$, and the appearance of PEG proton peaks at around 3.3-3.9 ppm, with the expected intensities, confirm the completion of the alkyneazide click reaction (Fig. 1 and S24†). The GPC molecular weight $\left(M_{\mathrm{n}}\right)$ of the PEG-jacketed HBP increased to $\sim 4800 \mathrm{~g}$ $\mathrm{mol}^{-1}$ with a slightly smaller PDI of 2.3 (Fig. S22 $\dagger$ ).

Likewise, the peripheral propargyl groups of the HBP were also clicked with a hydrophobic segment, namely docosyl (DOCO) azide, under similar reaction conditions (Scheme 3). The ${ }^{1} \mathrm{H}-\mathrm{NMR}$ spectra of the clicked HBPs (Fig. 1) again reveal the completion of the click reaction, as evident from the complete disappearance of propargyl protons peaks, the appearance of the triazole ring proton peak, and the appearance of peaks associate with the clicked segments; the peaks are in the expected intensities (Fig. 1 and S24 $\dagger$ ). The GPC molecular weight $\left(M_{\mathrm{n}}\right)$ of the DOCO-jacketed HBP increased to $\sim 4300 \mathrm{~g} \mathrm{~mol}^{-1}$ with a slightly larger PDI of 4.8 (Fig. S23†).

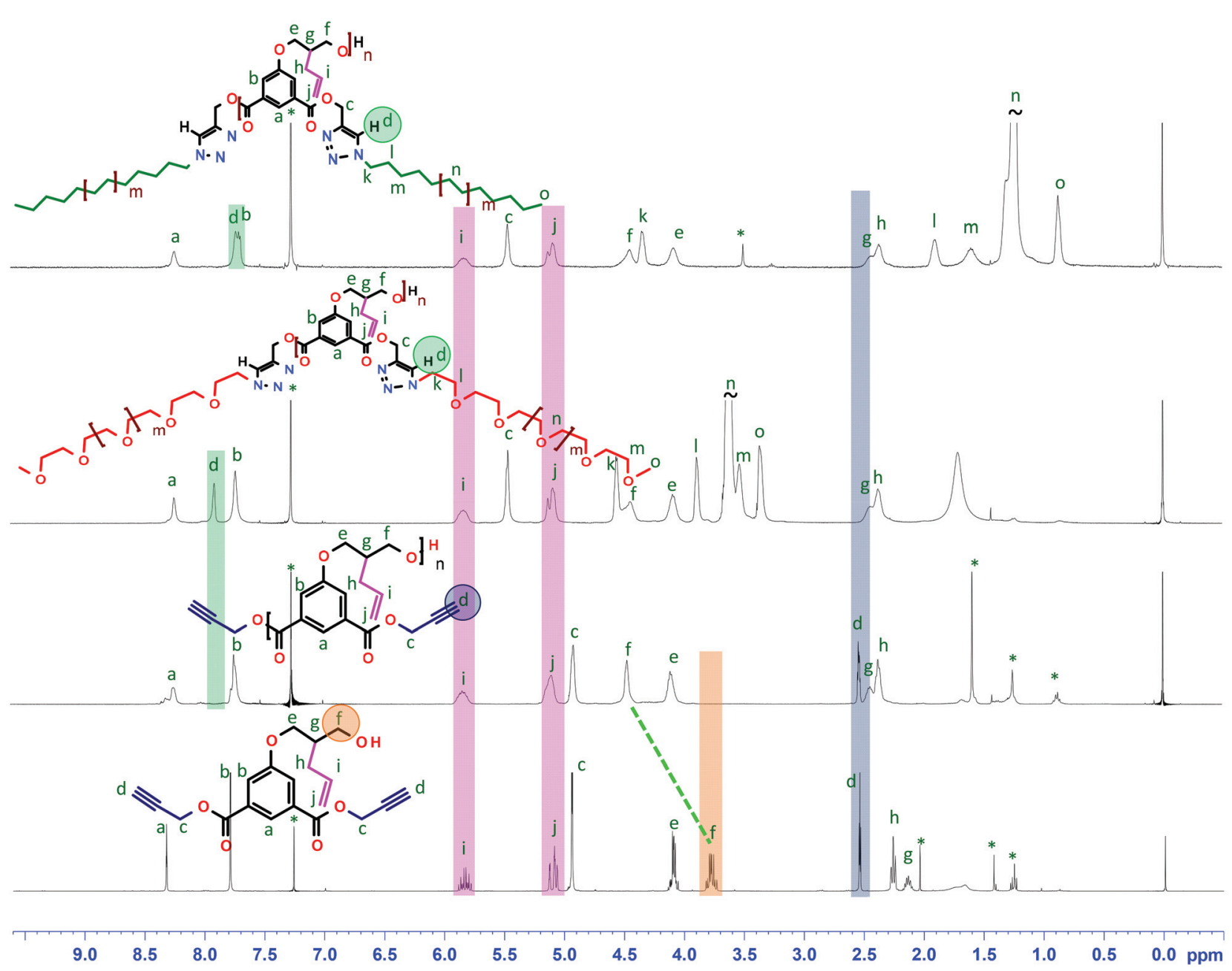

Fig. $1{ }^{1} \mathrm{H}$-NMR spectra (in $\mathrm{CDCl}_{3}$ ) of the monomer, parent $\mathrm{HBP}$, and those peripherally clicked with either PEG350-azide or docosyl azide. The grey band reveals the disappearance of the propargyl peak and the green band reveals the appearance of the triazole peaks. The peaks marked by an asterisk $\left(^{*}\right)$ are due to solvents or DBTDL catalyst. For further information concerning peak intensities refer to Fig. S24. $\dagger$ 

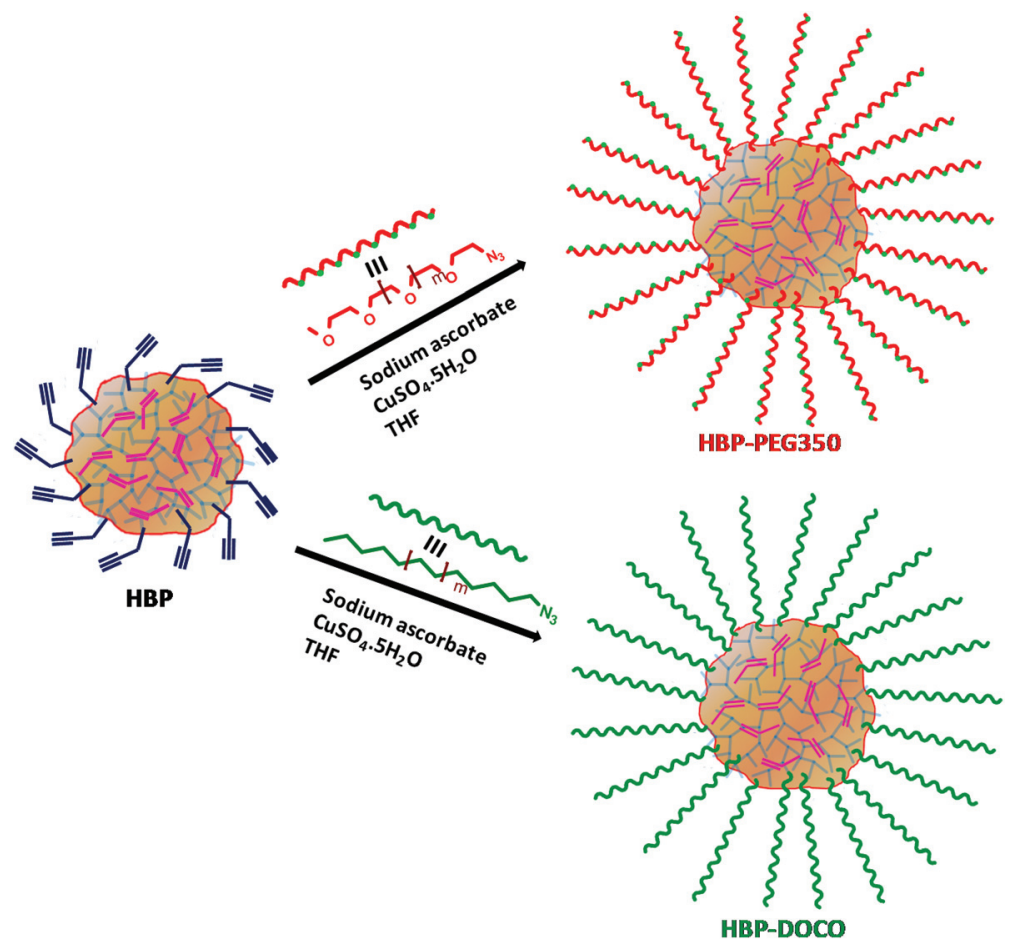

Scheme 3 Schematic representation of selective functionalization of periphery of the HBP with either PEG350 azide or docosyl azide.

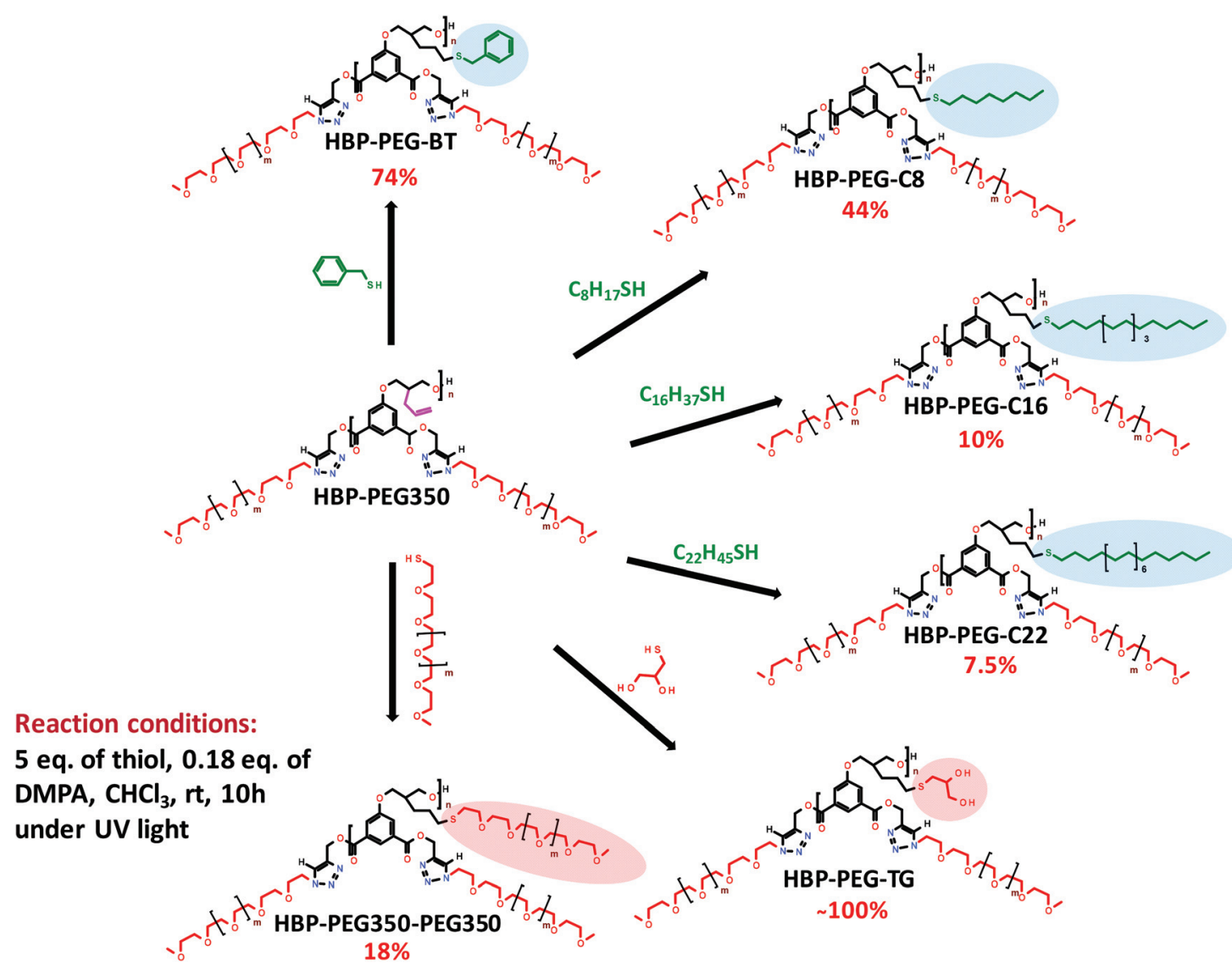

Scheme 4 Reaction of core allyl groups of the PEG-jacketed HB polyesters with hydrophobic thiols and hydrophilic thiols of varying size in chloroform; the extent of reaction (number shown below each structure) is seen to decrease with the size of the reacting organic thiol. 


\section{Core functionalization of peripherally PEGylated HBPs}

The peripherally functionalized HBPs may be viewed as jacketed HBPs, since their solubility depends primarily on the nature of the peripheral segments; the PEG jacketed HBP was soluble in polar solvents, like methanol, whereas the DOCOjacketed HBP was soluble in nonpolar solvents, like toluene, although both were readily soluble in solvents, like THF and chloroform. It is important to recognize that, unlike dendrimers, HBPs do not possess a discrete core-shell structure; however, once jacketed with long peripheral segments, they could behave like core-shell structures. Despite the large number of linear defects present in HBPs, once all the terminal propargyl groups are clicked with long chain alkyl (or PEG) azide, the conformation of the jacketed HBPs in solution, especially in a selective solvent, would resemble a core-shell type system, with the peripheral segments forming the shell and the HBP backbone the core. Such conformational reorganization and segregation of the peripheral segments from those of the backbone is revealed from both the bulk morphology and solution self-assembly studies. Earlier solution studies on different types of HBPs have demonstrated the ability of peripherally functionalized amphiphilic
HBPs to form unimolecular micelles and reverse micelles, and also form a variety of assemblies resulting from the core-shell segregation; ${ }^{34-37}$ furthermore, the importance of the hyperbranched topology on the ability to segregate the core and shell domains was examined by careful comparison with linear analogues. ${ }^{38}$ Thus, despite the randomly branched structure, suitably derivatized amphiphilic HBPs can exhibit core-shell segregation; more so in segment-selective solvents. To understand the factors that govern the access of the internal allyl groups to reaction with thiols in our peripherally jackets derivatives, the PEGylated HBP was first reacted with hydrophobic thiols of different sizes via the thiol-ene reaction (Scheme 4).

First, four thiols, namely benzyl thiol (BT), octane thiol (C8), hexadecane thiol (C16) and docosane thiol (C22), were reacted with the PEGylated $\mathrm{HB}$ polyester; the reaction was carried out in chloroform for a fixed duration of $10 \mathrm{~h}$ at room temperature under UV irradiation (365 nm), in the presence of a photo-initiator, (2,2-dimethoxy-2-phenylacetophenone) (DMPA). The proton NMR spectra of the isolated polymers are shown in Fig. 2; the spectra clearly reveal that the peaks due to the allyl protons (5.1 ppm and $5.8 \mathrm{ppm}$ ) have not fully disappeared in all cases. From the relative intensity of the

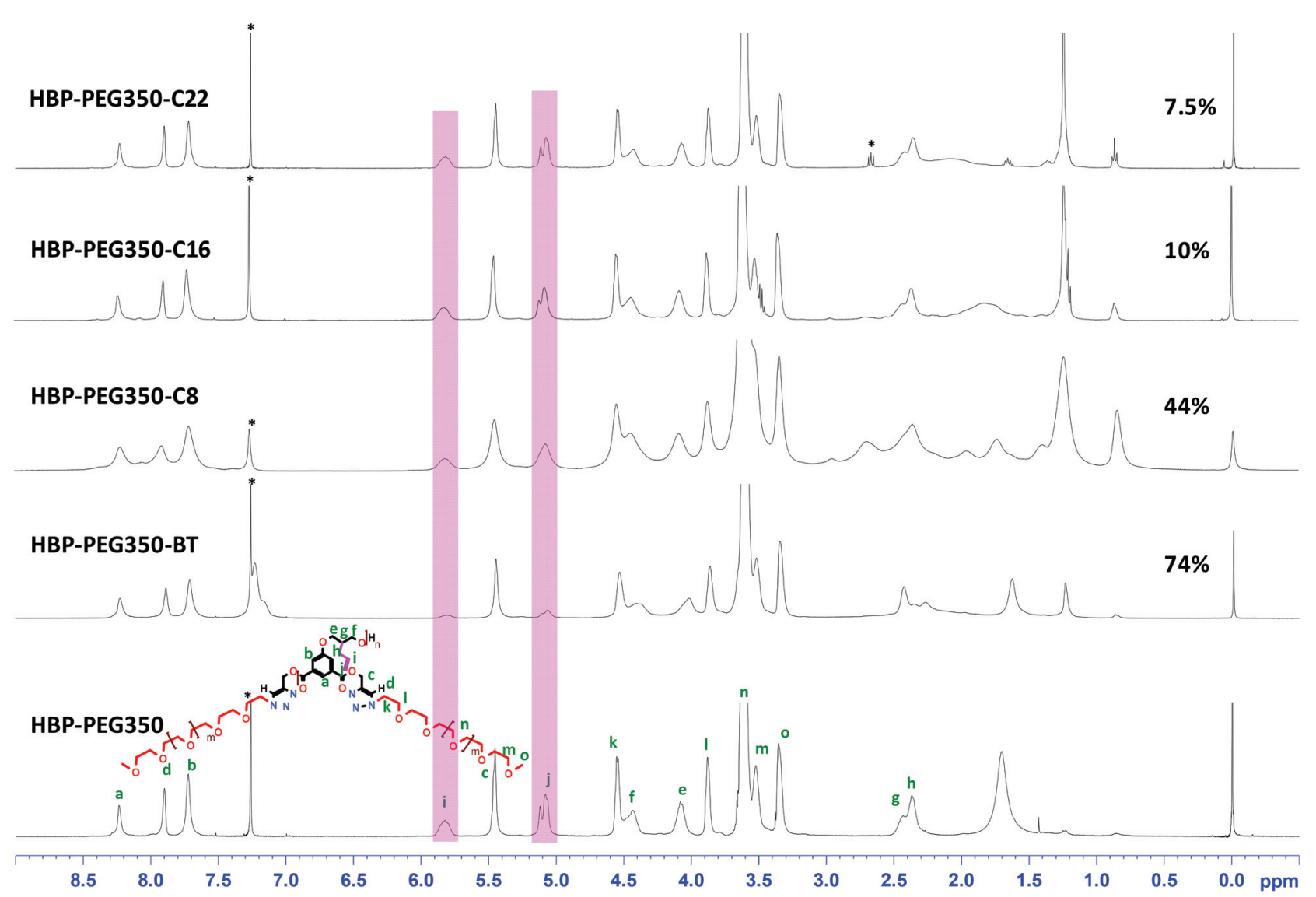

Fig. $2{ }^{1} \mathrm{H}$-NMR spectra (in $\mathrm{CDCl}_{3}$ ) of the core functionalization of peripherally PEGylated $\mathrm{HBP}$, along with the peak assignments. The intensity of the residual allyl peaks reveals the extent of reaction; the intensity of the peak at $\sim 5.5 \mathrm{ppm}$, due to the methylene protons adjacent to the triazole ring, was set to value of 2 and the relative intensity of the peak at $\sim 5.8 \mathrm{ppm}$ due to a single allyl proton was used to estimate the extent of reaction (see Fig. S25 † for details). The peaks marked by an asterisk $\left(^{*}\right)$ are due to solvents. 


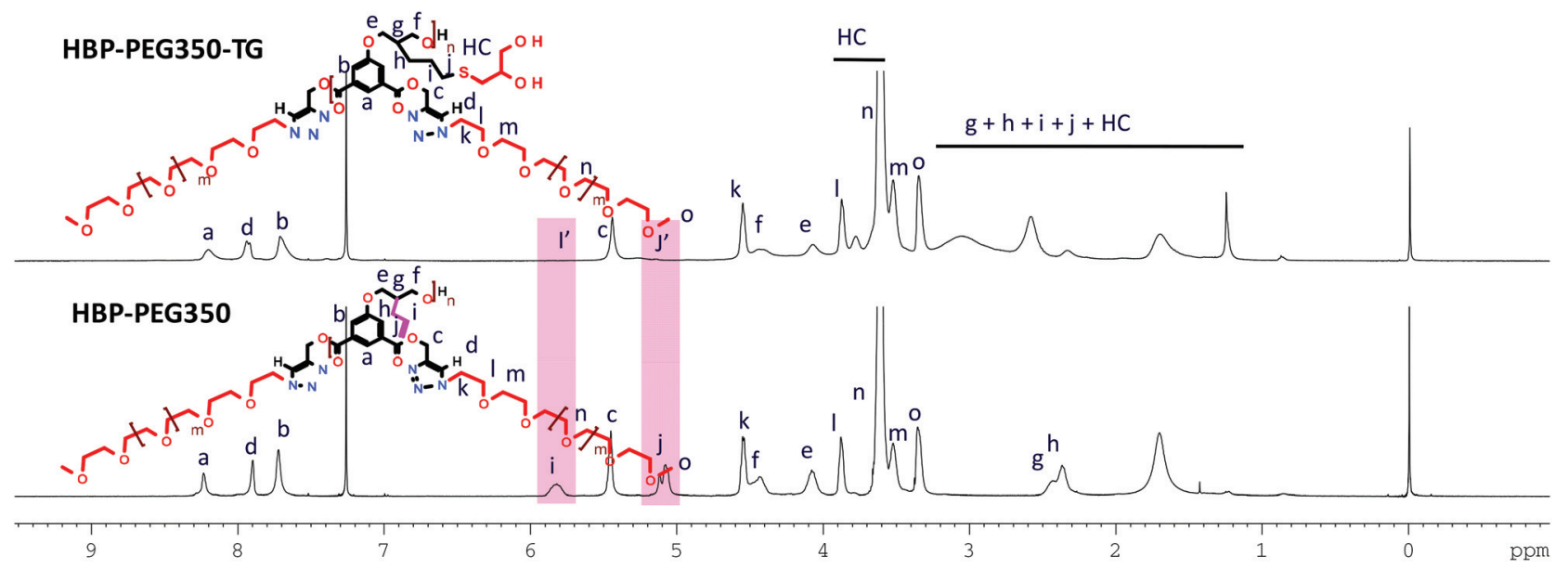

Fig. $3{ }^{1} \mathrm{H}$-NMR spectra (in $\mathrm{CDCl}_{3}$ ) of the core functionalized hydrophilic PEGylated HBP, along with peak assignments. The complete disappearance of the allyl peaks (shaded in pink) suggests quantitative reaction of the core in the case of thioglycerol.

residual allyl proton peaks, the extent of conversion was calculated, and these have been listed directly in the Fig. 2 .

From the conversion data, it is evident that smaller size thiols react more readily, whereas the larger size ones are unable to access the allylic sites within the PEG-jacketed coreregion of the HBP; the conversion in the cases of hexadecyl and docosyl thiols are below 10\%. Likewise, the PEGylated HB polyester was reacted with two hydrophilic thiols, namely thioglycerol and PEG350-thiol. From Fig. 3, it is evident that the reaction goes to $\sim 100 \%$ completion in the case of thioglycerol, a relatively small molecule, but in the case of PEG350-thiol, the extent of reaction was only $18 \%$ (Fig. S15†).

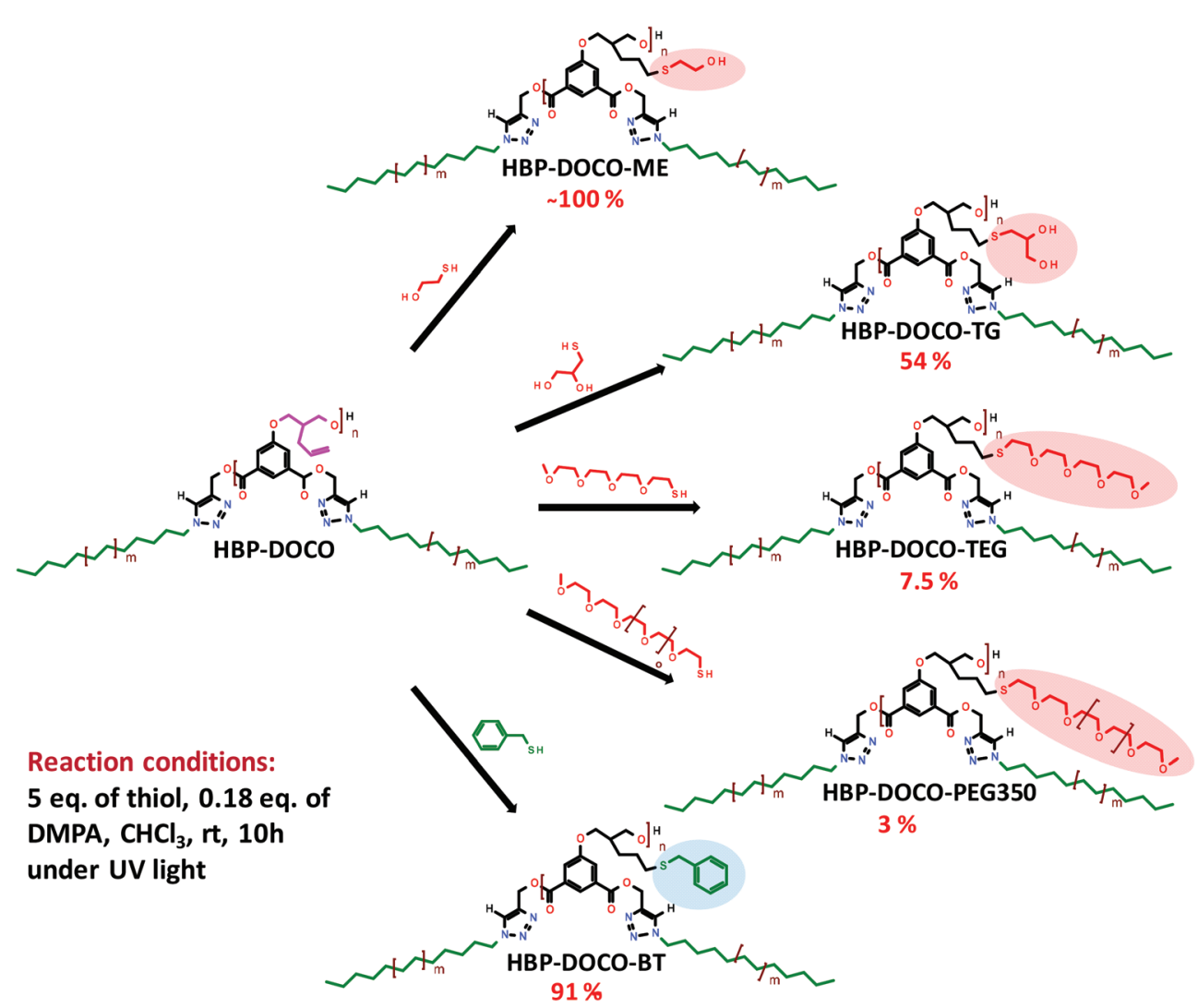

Scheme 5 Reaction of core allyl groups of the hydrophobically jacketed HB polyesters with hydrophobic thiol and hydrophilic thiols of varying size; the extent of reaction (number shown below each structure) is seen to decrease with the size of the reacting organic thiol. 


\section{Core-functionalization of hydrophobically jacketed HBPs}

To examine the access to internal allyl groups in the DOCOjacketed HB polyester, the hydrophobically modified HBP was first treated with a series of hydrophilic thiols of different sizes, namely 2-mercaptoethanol, thioglycerol, tetraethylene glycol monomethyl ether mono-thiol (TEG thiol) and polyethylene glycol 350 monomethyl ether mono-thiol (PEG350 thiol) (Scheme 5). The extent of reaction varied considerably with the size of the thiol; 2-mercaptoethanol reacted nearly quantitatively, whereas the extent of reaction was considerably lower, at only $54 \%$, in the case of thioglycerol, $7.5 \%$, in the case of TEG thiol, and only 3\%, in the case of PEG350 thiol (Fig. 4). However, when the hydrophobically modified HB polyester was reacted with benzyl thiol, the reaction proceeded to 91\% (Scheme 5 and Fig. 4); Table 1 provides a comparison of the extent of reaction of the hydrophilic PEGylated HB polyester and the hydrophobically jacketed HB polyester with the same pair of thiols. The data clearly reveal that the same thiol, such as thioglycerol, reacts completely in the case of PEGylated HBP, whereas the extent of reaction is only $54 \%$ in the case of
Table 1 Comparison of the extent of reaction of a hydrophobic (BT) and a hydrophilic thiol (TG) with the core allyl groups of hydrophilically and hydrophobically jacketed HBPs

\begin{tabular}{lll}
\hline Polymer & Thioglycerol (TG) & Benzyl thiol (BT) \\
\hline HBP-PEG350 & $\sim 100 \%$ & $74 \%$ \\
HBP-DOCO & $54 \%$ & $91 \%$
\end{tabular}

the hydrophobically modified DOCO-HBP; this clearly illustrates that both the size and polarity dictate the accessibility of peripherally jacketed HBPs. On the other hand, benzyl thiol reacts with the DOCO-jacketed HBP more readily that with the PEGylated derivative (91 versus $74 \%$, respectively). It is important to emphasize that the conversions do not represent limiting values but comparative ones, using different thiols, under identical reaction conditions. Thus, these globular HBPs suitably jacketed could serve as interesting scaffolds to install catalytic sites within the core; these internal sites could have preferential reactivity towards certain substrates over others.

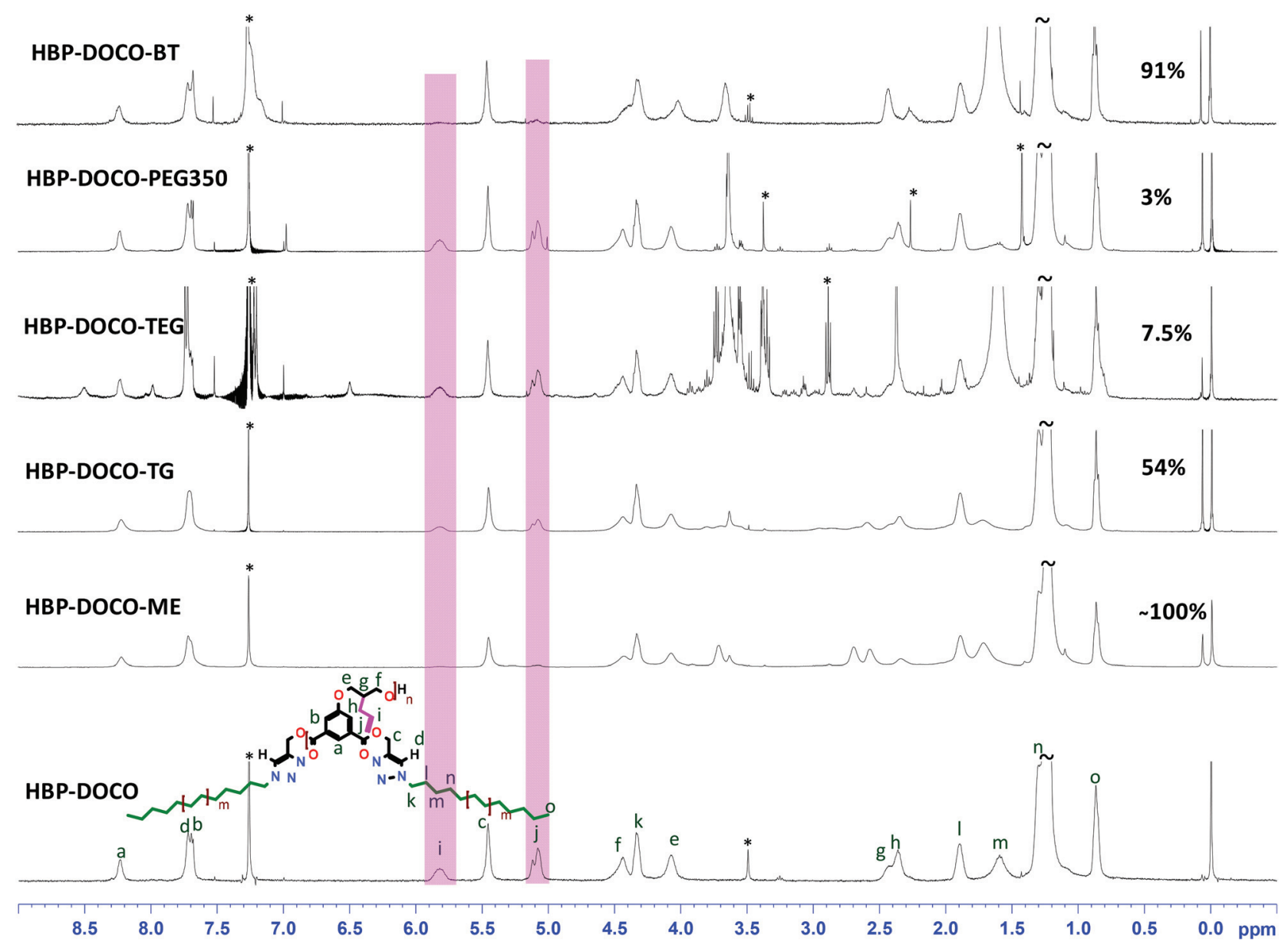

Fig. $4{ }^{1} \mathrm{H}$-NMR spectra (in $\mathrm{CDCl}_{3}$ ) of the core functionalization of peripherally hydrophobically jacketed DOCO-HBPs. The intensity of the residual allyl peaks reveals the extent of the reaction; as earlier, the relative intensities of the peaks at $\sim 5.5 \mathrm{ppm}$, due to the methylene protons adjacent to the triazole ring, and at $\sim 5.8 \mathrm{ppm}$ due to a single allyl proton was used to estimate the extent of reaction. The peaks marked by an asterisk (*) are due to solvents. For details concerning peak intensities refer Fig. S26. $\dagger$ 


\section{Conclusions}

In conclusion, a simple approach has been developed for the preparation of an orthogonally clickable HBP that carries an allyl group on every repeat unit and terminal propargyl groups on the periphery. Selective and quantitative functionalization of the peripheral propargyl groups via azide-alkyne click reaction was achieved to install either PEG350 or docosyl (C-22) segments on the periphery; access to the internally located allyl groups in these jacketed HBPS towards thiol-ene reaction was probed using a variety of thiols of different size and polarity. It was seen that as the size of the thiol increases the extent of reaction of the core allyl groups decreases; interestingly, apart from the size, the polarity of the thiol also influences the extent of reaction. Thus, whereas benzyl thiol reacts with the core allyl groups of the hydrophobically jacketed HBP to the extent of 91\%, thioglycerol transforms only about $54 \%$ of the allyl groups; on the other hand, in the case of PEG jacketed HBP, thioglycerol reacts quantitatively, whereas benzyl thiol reacts only to about $\sim 74 \%$. Although these reactions were carried out in a relatively good solvent, like $\mathrm{CHCl}_{3}$, that is expected to solvate both the core and peripheral segments, size and polarity of the reactant does appear to influence the conversion. Interestingly, despite the highly defective structure of HBPs, quantitative clicking of all the propargyl end groups, both on the terminal (T) and linear (L) units, with either long PEG or DOCO segments, appears to help segregate the peripheral segments from the backbone, thereby forming a core-shell type topology, which regulates the access to reactants. Thus, peripherally jacketed HBPs form an interesting class of globular structures wherein access, to internal sites can be regulated both by the size and relative polarity of the intruding reactant. This study provides some interesting directions that could be explored for the development of substrate-selective HBP-supported catalysts; work along these lines are currently ongoing.

\section{Conflicts of interest}

There are no conflicts to declare.

\section{Acknowledgements}

We would like to thank the Department of Science and Technology, New Delhi, for the research grant (SR/S1/OC-84/ 2012) and for the award of J C Bose fellowship (2011-2016 and 2016-2021) to SR. SKP would like to acknowledge MHRD for fellowship. We would like to thank Mr Arun Kumar Gayen for repeating some of the structural characterization of the samples and Professor Satish Patil and Mr Nilabja Maiti of SSCU department for their help with GPC measurements.

\section{References}

1 Hyperbranched Polymers: Synthesis, Properties, and Applications, ed. D. Yan, C. Gao and H. Frey, John Wiley \& Sons, Inc., New Jersey, 2011.

2 M. Seiler, Fluid Phase Equilib., 2006, 241, 155-174.

3 B. I. Voit and A. Lederer, Chem. Rev., 2009, 109, 59245973.

4 M. Irfan and M. Seiler, Ind. Eng. Chem. Res., 2010, 49, 1169-1196.

5 W. Jiang, Y. Zhou and D. Yan, Chem. Soc. Rev., 2015, 44, 3874-3889.

6 W. Wu, R. Tang, Q. Li and Z. Li, Chem. Soc. Rev., 2015, 44, 3997-4022.

7 Y. Zheng, S. Li, Z. Weng and C. Gao, Chem. Soc. Rev., 2015, 44, 4091-4130.

8 Y. Chen, M. Kuang, Z. Shen, J. Nieberle, H. Duan and H. Frey, Angew. Chem., Int. Ed., 2008, 47, 2227-2230.

9 Y. Liang, D. Wan, X. Cai, M. Jin and H. Pu, J. Polym. Sci., Part A: Polym. Chem., 2010, 48, 681-691.

10 S. Li, J. Han and C. Gao, Polym. Chem., 2013, 4, 1774-1787.

11 A. Saha and S. Ramakrishnan, Macromolecules, 2009, 42, 4028-4037.

12 A. Saha and S. Ramakrishnan, Macromolecules, 2009, 42, 4956-4959.

13 S. G. Ramkumar, K. A. Amala Rose and S. Ramakrishnan, J. Polym. Sci., Part A: Polym. Chem., 2010, 48, 3200-3208.

14 R. K. Roy and S. Ramakrishnan, J. Polym. Sci., Part A: Polym. Chem., 2011, 49, 1735-1744.

15 A. Z. Samuel and S. Ramakrishnan, Macromolecules, 2012, 45, 2348-2358.

16 A. Z. Samuel and S. Ramakrishnan, Langmuir, 2013, 29, 1245-1257.

17 N. S. Shree Varaprasad, P. Venkatesh and S. Ramakrishnan, J. Appl. Polym. Sci., 2014, 131, 40248.

18 S. K. Perala and S. Ramakrishnan, Macromolecules, 2017, 50, 8536-8543.

19 C. Santra, C. Kaittanis and J. M. Perez, Langmuir, 2010, 26, 5364-5373.

20 M. Calderón, M. A. Quadir, S. K. Sharma and R. Haag, Adv. Mater., 2010, 22, 190-218.

21 X. Yu, Z. Liu, J. Janzen, I. Chafeeva, S. Horte, W. Chen, R. K. Kainthan, J. N. Kizhakkedathu and D. E. Brooks, Nat. Mater., 2012, 11, 468-476.

22 M. Jikei and M. Kakimoto, Prog. Polym. Sci., 2001, 26, 12331285.

23 R. K. Roy and S. Ramakrishnan, J. Polym. Sci., Part A: Polym. Chem., 2013, 51, 4125-4135.

24 J. Han, S. Li, A. Tang and C. Gao, Macromolecules, 2012, 45, 4966-4977.

25 S. Li and C. Gao, Polym. Chem., 2013, 4, 4450-4460.

26 R. K. Roy and S. Ramakrishnan, Macromolecules, 2011, 44, 8398-8406.

27 For a recent review on HBP supported catalysis, see: K. Kirkorian, A. Ellis and L. J. Twyman, Chem. Soc. Rev., 2012, 41, 6138-6159. 
28 A. P. H. J. Schenning, C. Elissen-Roman, J-W. Weener, M. W. P. L. Baars, S. J. van-der Gaast and E. W. Meijer, J. Am. Chem. Soc., 1998, 120, 8199-8208.

29 Y. S. Sweet, D. M. Hedstrand, R. Spinder and D. A. Tomalia, J. Mater. Chem., 1997, 7, 1199-1205.

30 M. Ornatska, K. N. Bergman, B. Rybak, S. Peleshanko and V. V. Tsukruk, Angew. Chem., Int. Ed., 2004, 43, 5246-5249.

31 M. Ornatska, S. Peleshanko, K. L. Genson, B. Rybak, K. N. Bergman and V. V. Tsukruk, J. Am. Chem. Soc., 2004, 126, 9675-9684.

32 A. Ellis and L. J. Twyman, Macromolecules, 2013, 46, 7055-7074.
33 V. V. Rostovtsev, L. G. Green, V. V. Fokin and K. B. Sharpless, Angew. Chem., Int. Ed., 2002, 41, 25962599.

34 D. Y. Yan, Y. F. Zhou and J. Hou, Science, 2004, 303, 65.

35 H. Turk, A. Shukla, P. C. A. Rodrigues, H. Rehage and R. Haag, Chem. - Eur. J., 2007, 13, 4187-4196.

36 H. Cheng, S. Wang, J. Yang, Y. Zhou and D. Yan, J. Colloid Interface Sci., 2009, 337, 278-284.

37 Y. F. Zhou and D. Y. Yan, Chem. Commun., 2009, 1172.

38 Y. Chen, Z. Shen, L. Pastor-Perez, H. Frey and S.-E. Stiriba, Macromolecules, 2005, 38, 227-229. 